\title{
One health initiative to uncover epidemiology of melioidosis in southern Thailand
}

\author{
A Tuanyok
}

Melioidosis is endemic in most parts of Thailand. However, the prevalence of melioidosis in humans and animals and the occurrence of its pathogen, B.pseudomallei, in the natural environment of southern Thailand have not been updated for long time. Our goal is to develop a "One Health" initiative for melioidosis investigation and promote multidisciplinary melioidosis research in southern Thailand. As a part of this initiative we have been collecting $B$. pseudomallei isolates from human and animal cases and soils in Songkhla Province since January 2014. All isolates from patients admitted to three tertiary care hospitals including Songklanagarind Hospital, Hatyai Medical Center, and Songkhla Provincial Hospital were sent to a melioidosis laboratory at Prince of Songkla University for species confirmation. Molecular diagnostics using real-time PCR such as TTS-1, BTFC\&YLF, and LPS typing assays were used to identify B. pseudomallei. In addition, we have investigated the presence of $B$. pseudomallei in soils, especially in goat farms and a local zoo where animal cases have been reported. We used standard soil culturing techniques with selective media, Ashdown's agar and TBSS-50 broth, for B. pseudomallei isolation.

Suspected bacterial colonies grown on Ashdown's agar were subjected to further identification by latex agglutination, lateral flow immunoassay (LFI) and real-time PCR. The project is ongoing and so far we have confirmed at least 158 melioidosis cases from humans as well as the presence of $B$. pseudomallei in soils in Songkhla and nearby provinces. The infections were mostly seasonal and associated with rainfall. Genetic analysis using multi-locus sequencing typing (MLST) has indicated that most of these recent isolates had the same STs as those from Finkelstein's historic collection from southern Thailand a half century ago. Specifically, strains with STs 288, 1323, and 1359 were frequently found in Songkhla. Interestingly, at least 6 patients were confirmed to be infected by more than one sequence type. This suggests a high genetic diversity of $B$. pseudomallei in natural sources. Strains with ST3 were found in human and animal cases as well as in the environment. Collectively, we believe that our "One Health" initiative of melioidosis would form an integral part of regional threat assessment of Thailand and Southeast Asia.

Department of Infectious Diseases and Immunology, College of Veterinary Medicine, University of Florida, USA.

Address for correspondence: Dr Apichai Tuanyok, Department of Infectious Diseases and Immunology, College of Veterinary Medicine, University of Florida, USA. +1 3522948269 Email:tuanyok@ufl.edu

(D) https://orcid.org/0000-0002-3380-0448 